\title{
Effect of Foot Self-Care Behavior According to Severity of Diabetic Neuropathy in Japanese Patients with Diabetes
}

\author{
Masuko Sumikawa1*, Yumi Kuwamura2, Yasuyuki Sumikawa3 ${ }^{3}$ Toshiko Fujiwara4, \\ Chizuru Kamiya ${ }^{5}$
}

\author{
${ }^{1}$ Department of Nursing, School of Health Sciences, Sapporo Medical University, Sapporo, Japan \\ ${ }^{2}$ Department of Women's Health Nursing, Institute of Biomedical Sciences, Tokushima University Graduate School, \\ Tokushima, Japan \\ ${ }^{3}$ Department of Dermatology, School of Medicine, Sapporo Medical University, Sapporo, Japan \\ ${ }^{4}$ Sapporo Medical University Hospital, Sapporo, Japan \\ ${ }^{5}$ Kansai University of Nursing and Health Sciences, Awaji, Japan \\ Email: *masuko0811@sapmed.ac.jp
}

How to cite this paper: Sumikawa, M., Kuwamura, Y., Sumikawa, Y., Fujiwara, T. and Kamiya, C. (2018) Effect of Foot Self-Care Behavior According to Severity of Diabetic Neuropathy in Japanese Patients with Diabetes. Health, 10, 1192-1199.

https://doi.org/10.4236/health.2018.109091

Received: August 24, 2018

Accepted: September 11, 2018

Published: September 14, 2018

Copyright $\odot 2018$ by authors and Scientific Research Publishing Inc. This work is licensed under the Creative Commons Attribution International License (CC BY 4.0).

http://creativecommons.org/licenses/by/4.0/

\section{(c) (i) Open Access}

\begin{abstract}
PURPOSE: Diabetic patients with severe diabetic neuropathy often have hypoesthesia and numbness. This study evaluated foot self-care behavior according to severity of diabetic neuropathy. METHODS: We used a hand-held nerve conduction test device DPN check (HDN-1000, Omron) to evaluate severity of diabetic neuropathy. Foot self-care was evaluated using the Japanese SDSCA (the Summary of Diabetes Self-Care Activities measure). Foot self-care comprised visual inspection, washing, wiping interdigital areas, and checking shoes, and was scored according to the number of days self-care which was performed in the previous week. RESULTS: The study evaluated 103 diabetic patients (age: 65.7 years, diabetes duration: 13.9 years, HbA1c: $7.3 \%$ ). Total scores (out of 28 points) for self-care behavior according to neuropathy severity were 11.8 (Normal: $\mathrm{n}=54$ ), 10.6 (Mild: $\mathrm{n}=27), 13.3$ (Moderate: $\mathrm{n}=17$ ), and 10.8 (Severe: $\mathrm{n}=$ 5 ). Foot self-care scores were low in all groups, with particularly low scores in those with severe neuropathy. However, no statistically significant differences were observed. CONCLUSIONS: Foot self-care is essential in diabetes because lesions are more likely to occur in severe neuropathy. Living alone and the presence of recurrent foot lesions are associated with poor survival prognosis. Accordingly, foot-care intervention must take neuropathy severity and lifestyle factors into account. The severity of diabetic neuropathy must be determined and foot-care intervention should take lifestyle factors into account.
\end{abstract}

\section{Keywords}

Foot Self-Care Behavior, Diabetic Patients, Diabetic Neuropathy, Nerve 
Conduction Velocity, Nerve Conduction Amplitude

\section{Introduction}

Diabetic foot is characterized by infection, ulceration, or destruction of foot tissues in people with diabetes and is associated with neuropathy and/or peripheral artery disease [1]. Diabetic foot can also develop from several other conditions such as hypoesthesia and deformation caused by nerve disorder, dry skin and hyperkeratosis, or a decrease in peripheral artery blood flow. Moreover, infections can worsen the condition and can lead to amputation of the diseased limb [1] [2]. Previous reports have shown that lower limb amputation worsens patients' quality of life, and that the survival rates of diabetes patients with amputated lower limbs are $69.4 \%$ at one year and $30.9 \%$ at five years. Moreover, the report also showed that the survival rates of diabetes patients with end stage kidney disease are $51.9 \%$ at one year and $14.4 \%$ at five years [3].

Nerve disorder is the most frequent complication of diabetes. In previous studies, rates of nerve disorder vary because each study is based on different criteria. In Japan, a large research study has shown that the rate of patients diagnosed with diabetic nerve disorder was $47.1 \%$, and the frequency of asymptomatic nerve disorder was $40.3 \%$ [4]. This indicates that the early recognition of nerve disorder which can lead to foot ulcer among diabetes patients is very important. Previous studies have recommended measuring both nerve conduction velocity (NCV) and nerve conduction amplitude (NCA) [5] [6]. As the patients of severe diabetic nerve disorder often feel hypoesthesia and numbness, the foot care for these patients in daily life is very important. Although several reports have examined foot care activities in diabetes patients, to our knowledge, no studies have reported examining foot care activities in relation to the severity of peripheral nerve disorder [7] [8] [9] [10]. In this study, we examine diabetes patients' foot care activity according to the severity of diabetic nerve disorder. This research will evaluate foot care activities of diabetes patients who have skin integrity disorder or who are at risk for skin integrity disorder.

\section{Methods}

\subsection{Participants of the Study}

Patients with diabetes, are either attending or hospitalized in Hospital A. The data were collected from July 2015 to April 2016.

\subsection{Procedure for Data Collection}

We used a hand-held nerve conduction test device, the DPN check (HDN-1000, FUKUDA COLIN), to evaluate the severity of diabetic neuropathy. DPN Check easily assesses both the nerve conduction velocity (NCV) and nerve conduction amplitude (NCA) of the purely sensory sural nerve. Previous research has shown 
that the lower limit of normal NCV is $40 \mathrm{~m} / \mathrm{s}$ and the lower limit of normal NCA is $5-10 \mu \mathrm{V}$ in sural nerve [11].

Each foot of patients was measured by DPN check after 30 minutes rest. It took 5 minutes to measure. In addition, by adjusting for age, gender, and height, NCV and NCA values can be used to classify the severity of neuropathy into four levels: normal, mild, moderate, and severe. If the severity of nerve disorder was different between the right limb and left limb, then the data of the limb with the greatest severity were used to categorize patients into severity group.

Foot self-care behavior was evaluated using the Japanese SDSCA (the Summary of Diabetes Self-Care Activities measure) [12]. Foot self-care comprised 1) checking feet, 2) washing feet, 3) wiping interdigital areas, and 4) checking shoes. It was scored according to the number of days (a maximum of 7 days/activity) that self-care was performed in the previous week. These activities are recommended for all patients despite the severity of their neuropathy.

\subsection{Analysis of Data}

Descriptive statistics techniques were used to estimate the mean and frequencies and to examine normality. The Mann-Whitney $U$ test was used to examine the data between the two groups. The Kruskal-Wallis test was used to examine NCV, NCA, and foot-care behavior score data among the groups. The statistical software used IBM SPSS Statistics version 23.0. The level of statistical significance was set at 0.05

\subsection{Ethical Considerations}

This study was conducted with the approval of the Clinical Research Ethics Review of the Sapporo Medical University Hospital (approval number; 272-5).

\section{Results}

\subsection{Characteristics of the Patients Included in This Study (Table 1)}

A total of 103 diabetes patients who were either attending or hospitalized in Hospital A were included in this study. The average age of the patients was 65.7 \pm 10.4 years, and the average number of years with diabetes was $13.9 \pm 9.7$ years. The proportion of patients who self-reported neuropathy was $9.7 \%$. Table 1 provides the demographics and characteristics of the study participants.

\subsection{Results of NCV, NCA and Four Severities of Neuropathy Levels (Table 2)}

Table 2 provides the details of the NCV and NCA measures for the study participants. The average NCV of the patients was $48.7 \pm 6.4 \mathrm{~m} / \mathrm{s}$ in the right foot and $49.5 \pm 6.8 \mathrm{~m} / \mathrm{s}$ in the left foot. The average NCA of the patients was $9.6 \pm 8.1$ $\mu \mathrm{V}$ for the right foot and $10.0 \pm 7.4 \mu \mathrm{V}$ for the left foot. Neuropathy severity was categorized as normal, mild, moderate, and severe with scores of 54 (52.4\%), 27 
(26.2\%), 17 (16.5\%), and 5 (4.9\%), respectively. If the NCA score is lower than 2 $\mu \mathrm{V}$ and the NCV score was considered nil, then neuropathy severity was determined as "severe".

NCV and NCA were analyzed by sex and severity of nerve disorder. NCV was significantly lower in men than in women. NCV and NCA were significantly lower in the patient with severe nerve disorder compared to the other neuropathy categories.

\subsection{Foot Self-Care Behavior Scores and Four Severities of Neuropathy Levels (Table 3)}

Table 3 provides the details of the Foot self-care behavior scores and four severities of neuropathy levels. The average total foot self-care behavior score was $11.7 \pm 6.8$ (MAX: 28). The average score for each foot self-care activity was $3.6 \pm$ 3.3 for checking feet, $5.1 \pm 2.1$ for washing feet, $1.7 \pm 2.8$ for wiping interdigital areas, and $1.2 \pm 2.7$ for checking shoes (MAX: 7).

Table 1. Demographics and characteristics of the patients included in this study.

\begin{tabular}{|c|c|c|c|c|c|}
\hline & & $\mathrm{n}$ & $\%$ & Mean \pm SD & Range \\
\hline Age (years) & & 103 & 100 & $65.7 \pm 10.4$ & $31-85$ \\
\hline \multirow[t]{2}{*}{ Sex } & Male & 62 & 60.2 & & \\
\hline & Female & 41 & 39.8 & & \\
\hline \multirow[t]{3}{*}{ Diabetes type } & Type 1 & 11 & 10.7 & & \\
\hline & Type 2 & 87 & 84.8 & & \\
\hline & Other & 5 & 4.9 & & \\
\hline Diabetes duration (years) & & & & $13.9 \pm 9.7$ & $0-40$ \\
\hline $\mathrm{BMI}\left(\mathrm{kg} / \mathrm{m}^{2}\right)$ & & & & $24.3 \pm 3.2$ & $17.6-33.4$ \\
\hline Height $(\mathrm{cm})$ & & & & $161.5 \pm 7.9$ & $142-179$ \\
\hline HbAlc (\%) & & & & $7.3 \pm 1.4$ & $5.7-14.7$ \\
\hline \multirow[t]{5}{*}{ Nephropathy } & Stage 1 & 66 & 64.1 & & \\
\hline & Stage 2 & 21 & 20.4 & & \\
\hline & Stage 3 & 12 & 11.7 & & \\
\hline & Stage 4 & 3 & 2.9 & & \\
\hline & Unknown & 1 & 1 & & \\
\hline \multirow[t]{5}{*}{ Retinopathy } & NDR & 83 & 80.6 & & \\
\hline & SDR & 5 & 4.9 & & \\
\hline & PPDR & 2 & 1.9 & & \\
\hline & PDR & 12 & 11.7 & & \\
\hline & Unknown & 1 & 1 & & \\
\hline \multirow[t]{2}{*}{ Neuropathy (Patient self-report) } & No & 93 & 90.3 & & \\
\hline & Yes & 10 & 9.7 & & \\
\hline
\end{tabular}




\section{Continued}

\begin{tabular}{cccc}
\hline Numbness & Yes & 28 & 27.2 \\
Hypoesthesia & Yes & 17 & 16.5 \\
Dysesthesia & Yes & 14 & 13.6 \\
Leg cramps & Yes & 14 & 13.6 \\
The main skin symptoms & & & \\
Dry skin & Presence & 26 & 25.2 \\
Callus & Presence & 9 & 8.7 \\
Trichophytosis & Presence & 48 & 46.6 \\
\hline
\end{tabular}

BMI: Body Mass Index; NDR: no diabetic retinopathy; SDR: simple diabetic retinopathy; PPDR: preproliferative diabetic retinopathy; PDR: proliferative Diabetic Retinopathy.

Table 2. NCV and NCA by DPN check.

\begin{tabular}{cccccccccc}
\hline & \multicolumn{9}{c}{ NCV $(\mathrm{m} / \mathrm{s})$} \\
& $\mathrm{n}$ & $\%$ & Right side & $\mathrm{n}$ & Left side & $\mathrm{n}$ & Right side & $\mathrm{n}$ & Left side \\
\hline Total & 99 & 100 & $48.7 \pm 6.4$ & 95 & $49.5 \pm 6.8$ & 99 & $9.6 \pm 8.1$ & 97 & $10.0 \pm 7.4$ \\
Male & 59 & 59.6 & $47.0 \pm 5.6$ & 55 & $47.7 \pm 6.4$ & 59 & $9.7 \pm 8.7$ & 56 & $10.3 \pm 7.5$ \\
Female & 40 & 40.4 & $51.4 \pm 6.6$ & 40 & $52.0 \pm 6.6$ & 40 & $9.3 \pm 7.2$ & 41 & $9.6 \pm 7.2$ \\
Mann-Whitney U test & & & $\mathrm{p}=0.000$ & & $\mathrm{p}=0.001$ & & $\mathrm{P}=0.852$ & $\mathrm{p}=0.611$ \\
$\quad$ Normal & 54 & 52.4 & $51.5 \pm 3.4$ & 52 & $51.6 \pm 4.6$ & 54 & $10.6 \pm 8.6$ & 52 & $11.4 \pm 7.4$ \\
Mild neuropathy & 27 & 26.2 & $44.4 \pm 6.6$ & 26 & $47.5 \pm 7.6$ & 27 & $11.7 \pm 7.8$ & 27 & $10.4 \pm 8.0$ \\
Moderate neuropathy & 17 & 16.5 & $47.0 \pm 9.0$ & 15 & $46.1 \pm 9.6$ & 17 & $3.3 \pm 0.7$ & 16 & $5.6 \pm 3.8$ \\
Severe neuropathy & 1 & 4.9 & $46.0 \pm 0$ & 2 & $45.5 \pm 4.9$ & 1 & $4.0 \pm 0$ & 2 & $3.0 \pm 1.4$ \\
Kruskal-Wallis test & & & $\mathrm{P}=0.000$ & & $\mathrm{P}=0.015$ & & $\mathrm{P}=0.000$ & $\mathrm{P}=0.001$ \\
\hline
\end{tabular}

NCV: nerve conduction velocity; NCA: nerve conduction amplitude.

Table 3. Foot self-care behavior and Neuropathy levels.

\begin{tabular}{|c|c|c|c|c|c|c|c|}
\hline & $\mathrm{n}$ & $\%$ & 1) Checking feet & 2) Washing feet & $\begin{array}{l}\text { 3) Wiping } \\
\text { interdigital areas }\end{array}$ & $\begin{array}{l}\text { 4) Checking } \\
\text { shoes }\end{array}$ & $\begin{array}{c}\text { Total scores of } \\
1)-4)\end{array}$ \\
\hline Total & 103 & 100 & $3.6 \pm 3.3$ & $5.1 \pm 2.1$ & $1.7 \pm 2.8$ & $1.2 \pm 2.7$ & $11.7 \pm 6.8$ \\
\hline Normal & 54 & 52.4 & $3.4 \pm 3.4$ & $5.0 \pm 2.1$ & $2.0 \pm 2.9$ & $1.4 \pm 2.8$ & $11.8 \pm 7.7$ \\
\hline Mild neuropathy & 27 & 26.2 & $3.6 \pm 3.0$ & $5.3 \pm 1.9$ & $0.7 \pm 1.6$ & $1.1 \pm 2.5$ & $10.6 \pm 3.2$ \\
\hline Moderate neuropathy & 17 & 16.5 & $3.8 \pm 3.5$ & $5.6 \pm 2.4$ & $3.1 \pm 3.5$ & $0.8 \pm 2.3$ & $13.3 \pm 8.5$ \\
\hline Severe neuropathy & 5 & 4.9 & $5.2 \pm 2.7$ & $5.2 \pm 2.7$ & $0 \pm 0$ & $1.6 \pm 3.1$ & $10.8 \pm 4.8$ \\
\hline \multicolumn{3}{|c|}{ Kruskal-Wallis test } & $\mathrm{P}=0.596$ & $\mathrm{P}=0.345$ & $\mathrm{P}=0.043$ & $\mathrm{P}=0.659$ & $\mathrm{P}=0.809$ \\
\hline
\end{tabular}

The relationship between severity and subjective symptoms was analyzed. There were insignificant differences between severity and numbness, hypoesthesia, and dysesthesia $(\mathrm{P}=0.727, \mathrm{P}=0.953, \mathrm{P}=0.227)$.

The behavior score of wiping interdigital areas was significantly different according to severity of neuropathy $(\mathrm{P}=0.043)$. 


\section{Discussion}

In this study, the NCA and NCV of diabetes patients' sural nerve were measured and the relationship between severity of nerve disorder and foot care activities were analyzed. The average HbAlc of patients included in this study was 7.3\%, which is equal to that of Japanese diabetes patients. This suggested that the patients included in this study were general to patients in Japan.

The proportion of patients who self-reported neuropathy was $9.7 \%$; however, $47.6 \%$ of patients were evaluated as having mild to severe neurological disorder in this study. This indicated that many patients were not aware of their neuropathy. Identifying diabetic neuropathy at an early stage and performing appropriate foot care leads to the prevention of lower limb amputation. Moreover, it is necessary for patients to incorporate foot care into their everyday life and continue their self-management.

Scoring of foot care behavior revealed that checking feet, washing feet, wiping interdigital areas, and checking shoes were applicable to all diabetes patients. The checking of feet is important for the early detection of trauma. However, patients checked their feet only 3.6 times out of a week, but daily observation is necessary, especially for patients who are feeling impaired. Washing feet was done 5.1 times per week, which may be done as a Japanese bathing habit, but it is presumed that many patients can maintain skin cleanliness. However, the type of washing method was not investigated in this study, so it is unknown whether appropriate washing methods were used. For example, cleaning skin with alkaline soap can add to the original skin dryness in patients with autonomic neuropathy, leading to further reduction of sebum and lowering the barrier function of the skin [13]. Wiping interdigital areas had a low implementation rate at 1.7 times per week. Drying water between the toes after washing the foot is an action necessary to prevent tinea infection. Fungal infections were observed more frequently in patients with poor glycemic control [14]. The prevalence of tinea pedis is high in diabetes patients with poor glycemic control. It is said that diabetes patients have 2 times the tinea compared to non-diabetes patients [15]. Prevention and treatment of tinea is important because it can cause severe secondary infections. The implementation rate for checking shoes was low at 1.2 times per week. For patients with impaired sensation, something as simple as pebbles in the shoes can go unnoticed and lead to trauma.

The more complications diabetes patients develop, the more complicated the treatment becomes and the more items to be self-managed. It is important for patients to be able to incorporate self-care activities into their daily lives without difficulty, and it is necessary to carefully grasp the patient's psychological state. Living alone and the presence of recurrent foot lesions are associated with poor survival prognosis [16]. Also, as Japan is an aging society with a prevalence of diabetes, it is believed that it relates to dementia and depression so support for key persons such as families is indispensable [17]. Also, many living alone need to strengthen their cooperation with the Regional Comprehensive Support Center and visiting nurses. 


\section{Conclusion}

Foot self-care scores of diabetes patients were low in all groups, with particularly low scores in those with severe neuropathy. Foot self-care is essential in diabetes because lesions are more likely to occur in severe neuropathy. Living alone coupled with the presence of recurrent foot lesions is associated with poor survival prognosis. Thus, future foot-care interventions should take into account neuropathy severity and lifestyle factors.

\section{Acknowledgements}

The authors would like to thank the diabetic patients who participated in this research, as well as Dr. Shigeyuki Saito in Sapporo medical University, Dr. Hiroshi Akasaka in Osaka University Hospital, Dr. Minoru Kubota in Health care center Kwansei Gakuin. Without them, this study would not have possible.

This study was supported by a Grant-in-Aid for Scientific Research (C) from the Japan Society for the Promotion of Science (number 15K11591).

\section{Limitation of the Study}

For this study, it was unclear whether nurses interviewed patients directly about foot care behavior, so the original self-management status was declared. Also, because the proportion of people in each neuropathy category was not constant, sufficient statistical analysis could not be performed. It will be necessary to increase the number of participants in future studies. Also, in future studies, it would be beneficial to grasp the degree of neuropathy from an early stage and to develop the contents of foot care by severity and to develop an index for evaluating them.

\section{Conflicts of Interest}

The authors declare no conflicts of interest regarding the publication of this paper.

\section{References}

[1] The International Working Group on the Diabetic Foot (IWGDF) (2015) IWGDF Guidance on the Diagnosis and Management of Foot Infections in Persons with Diabetes. Prepared by the IWGDF Working Group on Foot Infections. http://www.iwgdf.org/files/2015/website_infection.pdf

[2] Frylberg, R.G., Zgonis, T., Armstrongs, D.G., et al. (2006) Diabetic Foot Disorders: A Clinical Practice Guideline (2006 Revision). The Journal of Foot Ankle Surgery, 45, S1-S66. https://doi.org/10.1016/S1067-2516(07)60001-5

[3] Aulivola, B., Hile, C.N., Hamdan, A.D., Sheahan, M.G., Veraldi, J.R., Skillman, J.J., Campbell, D.R., Scovell, S.D., LoGerfo, F.W. and Pomposelli Jr., F.B. (2004) Major Lower Extremity Amputation: Outcome of a Modern Series. Archives of Surgery, 139, 395-399. https://doi.org/10.1001/archsurg.139.4.395

[4] Japan Promotion Council for Diabetes Prevention and Countermeasures (2008) Report on Abnormalities in Foot Appearance and Diabetic Neuropathy in Diabetes 
Patients in Japan. Japanese Medical Association, Tokyo, Japan. (In Japanese) http://dl.med.or.jp/dl-med/tounyoubyou/diabetes080312.pdf

[5] Shimada, H., Miki, T., Okuno, Y., Nishizawa, Y. and Morii, H. (1998) Clinical Significance of Evaluating the Sensory Nerve Action Potential in Diabetic Polyneuropathy. Journal of the Japan Diabetes Society, 41, 23-27. (In Japanese)

[6] Yanagida, H., Ando, A., Kenta, O., Nagasaka, S., Ishibashi, S., Kotanai, K., Hasegawa, O. and Taniguchi, N. (2015) Determination of Reference Ranges for Nerve Conduction Studies: Influence of Age, Height and Gender. Jichi Medical University Journal, 38, 27-39. (In Japanese)

[7] Daitoku, M., Egawa, T., Fujiwara, Y. and Okumiya, A. (2007) Effects of Foot Care Intervention on Self-Care Behavior in the Patients with Diabetes Mellitus. Journal of the Japan Diabetes Society, 50, 163-172. (In Japanese)

[8] Yamada, R., Inagaki, M., Kitade, Y., Furuya, K., Tsuda, S., Ito, H., Nishizawa, M., Nakagawa, A., Nakano, S. and Furuya, D. (2012) Influences of a Novel Foot Care Education Program for Keeping up Moisture of Skin on the Recognition and the Action to Diabetic Foot. Journal of the Japan Diabetes Society, 55, 392-397. (In Japanese)

[9] Hayashino, Y. and Ishii, H. (2016) The Relationship between Patient Perception of Healthcare Provision by Professionals and the Self-Care Activity of Patients with Diabetes: Japanese Subgroup Analysis of the Second Diabetes Attitudes, Wishes, and Needs (DAWN2) Study. Diabetology International, 7, 111-118. https://doi.org/10.1007/s13340-015-0216-0

[10] Ahmad Sharoni, S.K., Minhat, H.S., MohdZulkefli, N.A. and Baharom, A. (2016) Health Education Programmes to Improve Foot Self-Care Practices and Foot Problems among Older People with Diabetes: A Systematic Review. International Journal of Older People Nursing, 11, 214-239. https://doi.org/10.1111/opn.12112

[11] Araki, E and Nakamura, J. (Eds.) (2013) Diabetic Neuropathy. Nakayama Shoten Co., Ltd., Tokyo, 116-122. (In Japanese)

[12] Daitoku, M., Honda, I., Okumiya, A., Yamasaki, Y., Kasayama, S., Ikegami, H., Miyagawa, J., Kubota, M. and Egawa, T. (2006) Validity and Reliability of the Japanese Translated "The Summary of Diabetes Self-care Activities Measure". Journal of the Japan Diabetes Society, 49, 1-9. (In Japanese)

[13] Schmid-Wendtner, M.H. and Korting, H.C. (2006) The pH of the Skin Surface and Its Impact on the Barrier Function. Skin Pharmacology and Physiology, 19, 296-302. https://doi.org/10.1159/000094670

[14] Akkus, G., Evran, M., Gungor, D., Karakas, M., Sert, M. and Tetiker, T. (2016) Tinea Pedis and Onychomycosis Frequency in Diabetes Mellitus Patients and Diabetic Foot Ulcers. A Cross Sectional-Observational Study. Pakistan Journal of Medical Sciences, 32, 891-895.

[15] Ogasawara, Y. (2003) Prevalence and Patient's Consciousness of Tinea Pedis and Onychomycosis. Japanese Journal of Medical Mycology, 44, 253-260. (In Japanese) https://doi.org/10.3314/jjmm.44.253

[16] Kurita, S., Kanamori, T., Ishikura, K. and Nagaoka, T. (2018) Clinical Characteristics of Diabetic Patients with Foot Ulcers and an Analysis of the Factors Affecting Their Prognosis. Journal of the Japan Diabetes Society, 61, 1-8. (In Japanese)

[17] Katon, W., Pedersen, H.S., Ribe, A.R., Fenger-Grøn, M., Davydow, D., Waldorff, F.B. and Vestergaard, M. (2015) Effect of Depression and Diabetes Mellitus on the Risk for Dementia: A National Population-Based Cohort Study. JAMA Psychiatry, 72, 612-619. https://doi.org/10.1001/jamapsychiatry.2015.0082 